\title{
Pharmacokinetics and tolerance study of intravitreal injection of dexamethasone-loaded nanoparticles in rabbits
}

This article was published in the following Dove Press journal:

International Journal of Nanomedicine

3 September 2009

Number of times this article has been viewed

\section{Linhua Zhang' \\ Yue $\mathrm{Li}^{2}$ \\ Chao Zhang' \\ Yusheng Wang ${ }^{2}$ \\ Cunxian Song'}

Institute of Biomedical Engineering, Peking Union Medical College and Chinese Academy of Medical Sciences, Tianjin, China; ${ }^{2}$ Department of Ophthalmology, Institute of Ophthalmology of Chinese PLA, Xijing Hospital, Fourth Military Medical University, Xi'an, China
Correspondence: Cunxian Song Institute of Biomedical Engineering, Peking Union Medical College and Chinese Academy of Medical Sciences, Tianjin 300I92, China

Tel +8602287892052

Email scxian@tom.com

\begin{abstract}
The aim of the study was to investigate the tolerance and pharmacokinetics of dexamethasone (DEX)-loaded poly(lactic acid-co-glycolic acid) nanoparticles (DEX-NPs) in rabbits after intravitreal injection. The DEX-NPs were prepared and characterized in terms of morphology, particle size and size distribution, encapsulation efficiency, and in vitro release. Ophthalmic investigations were performed, including fundus observation and photography, intraocular pressure measurement, and B-scan ocular ultrasonography. There were no abnormalities up to 50 days after administration of DEX-NPs in rabbits. The DEX concentrations in plasma and the ocular tissues such as the cornea, aqueous humor, lens, iris, vitreous humor, and chorioretina were determined by high-pressure liquid chromatography. The DEX-NPs maintained a sustained release of DEX for about 50 days in vitreous and provided relatively constant DEX levels for more than 30 days with a mean concentration of $3.85 \mathrm{mg} / \mathrm{L}^{-1}$. Based on the areas under the curve, the bioavailability of DEX in the experimental group was significantly higher than that in the control group injected with regular DEX. These results suggest that intravitreal injection of DEX-NPs lead to a sustained release of DEX with a high bioavailability, providing a basis for a novel approach to the treatment of posterior segment diseases.
\end{abstract}

Keywords: dexamethasone, nanoparticles, intravitreal injection, pharmacokinetics

\section{Introduction}

The leading cause of vision impairment and blindness is posterior segment diseases, including age-related macular degeneration, proliferative vitreoretinopathy, diabetic macular edema, and endophthalmitis. However, delivery of drugs to the posterior segment is challenging, due to the anatomic and physiologic barriers of the eye. ${ }^{1}$ Intravitreal injection has been reported to be the most direct therapeutic approach for delivering the drug directly into the posterior segment, bypassing the corneoscleral barriers. ${ }^{2}$ This type of administration is recommended when less aggressive routes are discarded or for severe pathologies that affect the vitreous or the retina. Intravitreal injections generally achieve improved drug absorption and diminish or prevent the adverse effects associated with systemic or topical treatment. However, many of the vitreoretinal diseases that intravitreal injections target are not controlled by a single injection, and the need for multiple injections is poorly tolerated and has inherent risks, such as endophthalmitis, cataract, retinal detachment, and vitreous hemorrhage. ${ }^{3,4}$ Moreover, the low therapeutic rate of some drugs used for the treatment of the posterior segment diseases can lead to toxic concentrations in the retina. Some of these inconveniences can be overcome by the use of new dosage forms such as controlled release systems, which allow a reduction in the number of injections, 
or surgical implantation of sustained drug release devices. Sustained drug release devices require surgical placement and are not ideal for the routine management of common posterior segment diseases due to the risk of surgery, unless the benefit is exceedingly high. ${ }^{5}$

The development of sustained release systems that can lead to a controlled release of the drug and are adapted to the intraocular route are very useful because it would be possible to achieve the same effect with a single injection as with multiple doses, above all for drugs with a short half-life. ${ }^{6}$ It has been shown that sustained intraocular drug concentrations can be realized through coupling the desired drug to liposomes, microparticles, or nanoparticles. Nanoparticle drug delivery has demonstrated promising results in ophthalmic drug delivery over the last 10 years. When delivered to the eye, these nanoparticles did not induce inflammatory reactions in the retinal tissue or disturb the organization of the surrounding ocular tissues. The major problem of intravitreal injection is inducing the stimulation of pathogenic immune responses, which results in photoreceptor degeneration. ${ }^{7-11}$ Various studies have also shown that intravitreal administration of nanoparticles did not generate organ-specific autoimmune phenomena. ${ }^{12}$

Dexamethasone (DEX) has demonstrated to be an efficient anti-inflammatory drug in the treatment of acute and chronic posterior segment eye diseases such as uveitis or affections that involve neovascularization, such as proliferative vitreoretinopathy or subretinal neovascularization. ${ }^{13}$ Current treatments of the posterior segment diseases using corticoids are performed by direct injections of corticoid solutions or suspensions. However, direct injections of corticoids into the vitreous often require large boluses and repeated injections to ensure therapeutic levels over an extended period of time, leading to a reduction of patient compliance, or to an increased likelihood of complications. ${ }^{14}$ The controlled-release delivery of DEX via a nanoparticle system would allow a sustained delivery of the drugs, thus prolonging the duration of drug action, avoiding the need for frequent intraocular injections, and decreasing the risk of complications.

Intravitreal injection of DEX-loaded nanoparticles for the posterior segment eye diseases requires a thorough understanding of its pharmacokinetics for further potential application. Although the pharmacokinetics and local distribution of DEX in the eye have been investigated in the past, ${ }^{15}$ we did not find information regarding its pharmacokinetics by intravitreal injection via a nanoparticle system. Taking the abovementioned information into account, it was hypothesized that DEX-loaded poly(lactic acid-co-glycolic acid) (PLGA) nanoparticles (DEX-NPs) could lead to controlled release of the drug following a single intravitreal injection and achieve the same effect as multiple injections of the conventional agent. To examine this hypothesis and evaluate the feasibility of intraocular application of drug-loaded nanoparticles, we have investigated the pharmacokinetic behavior and tolerance of DEX-NPs in ocular tissues and plasma after a single intravitreal injection in rabbits.

\section{Materials and methods \\ Materials}

Poly(lactic acid-co-glycolic acid) (PLGA) 50/50 (90,000 molecular weight) was purchased from Birmingham Polymers, Inc. (Birmingham, AL). Poly(vinyl alcohol) (PVA) with an average molecular weight of 30,000-70,000 was obtained from Sigma-Aldrich (St. Louis, MO). DEX was purchased from Sigma-Aldrich All organic solvents were either highpressure liquid chromatography (HPLC) or analytical grade.

\section{Nanoparticle preparation}

DEX-NPs were prepared by an oil-in-water $(\mathrm{O} / \mathrm{W})$ emulsion/ solvent evaporation protocol with minor modifications. ${ }^{16}$ Briefly, PLGA was dissolved in dichloromethane (DCM) and DEX in acetone. The two organic solutions were mixed and added to an aqueous solution containing 2.5\% PVA and sonicated over an ice bath using a microtip probe sonicator (Sonicator Model XL2020; Misonic, Inc., Farmingdale, NY) at $40 \mathrm{KW}$ power output for $10 \mathrm{~min}$ to form an $\mathrm{O} / \mathrm{W}$ emulsion. The organic solvents were allowed to evaporate overnight by stirring over a magnetic stir plate in a laminar flow cabinet. Nanoparticles thus formed were collected by ultracentrifugation, and then washed three times with distilled water to remove free drug and PVA. The final product was dried by lyophilization for $48 \mathrm{~h}$.

\section{Nanoparticle characterization}

Nanoparticle size distribution (mean diameter and polydispersity index) was determined by photon correlation spectroscopy (PCS) (Draft-290000AT; Brookhaven Co., Holtsville NY). The analysis was performed at a scattering angle of $90^{\circ}$ at a temperature of 25 using samples appropriately diluted with ultrapurified water. The morphology of the nanoparticles was ascertained by transmission electronic microscopy (TEM). A drop of the nanoparticle suspension was placed on the copper electron microscopy grids. The dried sample was then examined. The DEX content in the PLGA nanoparticles was assayed by HPLC. A few milligrams of nanoparticles were dissolved in chloroform, and then 
methanol was added to precipitate the polymer. The supernatant was obtained by centrifugation, diluted by methanol and the concentration of DEX in the supernatant was measured using HPLC. The HPLC system was equipped with a reverse phase Diamond $^{\circledR} \mathrm{C} 18$ column (inner diameter $150 \mathrm{~mm} \times 4.6 \mathrm{~mm}$, pore size $5 \mu \mathrm{m})$. The mobile phase consisted of $60 / 40(\mathrm{v} / \mathrm{v})$ methanol/water, which was degassed and vacuum filtered. The column was equilibrated before each run at a flow rate of $1.0 \mathrm{ml} / \mathrm{min}^{-1}$ and the temperature was thermostated at $35^{\circ} \mathrm{C}$ until a stable baseline was achieved. The compound was detected by a UV detector at $240 \mathrm{~nm}$ wavelength. Prednisone acetate-methanol solution was used as an internal standard. The encapsulation efficiency of DEX-NPs was calculated with the following equal formula:

Drug encapsulation efficiency $(\%, \mathrm{w} / \mathrm{w})=($ Actual drug loading/Theoretical drug loading) $\times 100$

\section{In vitro drug release}

In vitro release of DEX from the nanoparticles was performed in sodium phosphate-buffered saline (PBS; $\mathrm{pH} 7.4,0.1 \mathrm{M}$ ) at $37{ }^{\circ} \mathrm{C}$ with a dialysis membrane method on a shaker stand. The freeze-dried DEX-NPs were weighed, dispersed in $1 \mathrm{ml}$ of buffer and then put in dialysis bags (Sigma-Aldrich) with a molecular mass cut-off of 12,000 Da. Then the bag was put into a plastic tube immersed in $15 \mathrm{ml} \mathrm{PBS}$. The tube was placed in an incubator shaker, which was maintained at $37^{\circ} \mathrm{C}$ and shaken horizontally at $130 \mathrm{rpm}$. At predetermined time intervals, the release medium was completely withdrawn and replaced with an equal volume of fresh buffer. The concentration of DEX in the release medium was measured by HPLC as described above.

\section{Animal handling}

Forty-eight New Zealand White rabbits (NZW) of either sex, weighing 2 3 kg, were purchased from Tianjin Medical Laboratory Animals Center (Tianjin, China). The Administrative Committee on Animal Research in the Institute of Biomedical Engineering, Chinese Academy of Medical Science, approved all the protocols for animal experiments. All animal experiments were performed in compliance with the Guiding Principles for the Care and Use of Laboratory Animals, Peking Union Medical College of China. The animals were classified randomly into two groups: experimental group with thirty rabbits, and control group with eighteen rabbits. Before intravitreal injection, all of the eyes were found to be normal by fundus observation.

After being sterilized by $\gamma$-radiation, DEX-NPs were suspended in normal saline at the equivalent DEX concentration of $20 \mathrm{~g} / \mathrm{L}^{-1}$. Rabbits in the experimental group received intravitreal injections of $0.1 \mathrm{ml}$ of DEX-NPs suspension and the control rabbits received intravitreal injection of $0.1 \mathrm{ml} \mathrm{DEX}\left(20 \mathrm{~g} / \mathrm{L}^{-1}\right.$ in saline). For the experimental group, ophthalmic investigations, including fundus observation and photography, intraocular pressure (IOP) measurement, and B-scan ocular ultrasonography, were performed 1, 3, 7, $14,21,28,35,42,50$, and 80 days after injection. The drug concentrations in plasma and various ocular tissues, including the cornea, aqueous humor, lens, iris, vitreous, and chorioretina were measured by HPLC at each time point. For the control group, the same experiments were performed 1, 2, $3,5,7$, and 14 days after injection.

\section{Intravitreal injection and observation}

The animals were anesthetized by intramuscular injection of 0.3 0.4 ml Sumianxin mixture (dihydroetrophine, xylazole, haloperidol). The pupils were dilated with $2.5 \%$ phenylephrine hydrochloride. Paracentesis was performed with a 30-gauge needle attached to a tuberculin syringe before intravitreous injection. About $100 \mu \mathrm{l}$ of aqueous humor were withdrawn carefully from the eyes. The DEX-NPs or DEX alone $(100 \mu \mathrm{l})$ were injected into the midvitreous cavity with a 30-gauge needle on a 1-ml tuberculin syringe through the pars plana. The intravitreal injection was always carried out in both eyes for each rabbit. The eyes were examined with the indirect ophthalmoscope at each time point after injection; ophthalmic investigations including fundus observation and photography, IOP measurement, and B-scan ocular ultrasonography study were also performed at each time point.

\section{Preparation and purification of samples}

Three rabbits were randomly selected at each time point. Blood samples were taken from the marginal vein of the ear and collected in heparin tubes, and the plasma was taken for analysis after separation by centrifugation. Euthanasia was performed with an intracardiac pentobarbital overdose. Both eyes were quickly dissected under a microscope, and samples of each ocular tissue, including cornea, aqueous humor, lens, iris, vitreous, and chorioretina were taken and extracted for HPLC analyses. $0.1 \mathrm{ml}$ of PBS was added to the cornea, chorioretina, iris, and lens samples, and the mixture was homogenized in glass homogenizers. Prednisone acetate-methanol solution $(0.1 \mathrm{ml})$ was added to each sample as internal standard, then $2 \mathrm{ml}$ methyl tert-butyl ether (MTBE) was added to each solution. After vortex-mixing, the supernatant was dried by $\mathrm{N}_{2}$ purging and then dissolved in $0.1 \mathrm{ml}$ methanol for HPLC analysis as described above. 


\section{Preparation of calibration curves and data analysis}

Aliquots $(0.1 \mathrm{ml})$ of the internal standard and different concentrations of standard DEX were added to the rabbit plasma, and each ocular tissue, including the cornea, aqueous humor, lens, iris, vitreous, and chorioretina, to form a set of standard solutions. The samples were then prepared by the method described in 2.7 before HPLC analysis. Three injections $(20 \mu \mathrm{l})$ of each solution were made into the chromatographic system.

Calibration curves were constructed using the ratio of the peak area of DEX and internal standard by weighted $(1 / y)$ linear regression analysis. The concentrations of the samples obtained at each time point were calculated by refitting peak response ratios into the derived regression equation. Data were analyzed using SPSS software package (version 11.0 for Windows; SPSS Inc., Chicago, IL). The areas under the concentration versus time curves (AUC) values for DEX were calculated from the beginning to the end of the observation time.

\section{Statistical analysis}

Data were analyzed using SPSS software or Student's $t$-test when appropriate. Differences were considered to be significant at a level of $P<0.05$.

\section{Results}

\section{Characterization of DEX-NPs}

DEX-NPs were prepared using an emulsion/solvent evaporation protocol as described previously ${ }^{16}$ with minor modifications. TEM has been used in our research to characterize the morphology of DEX-NPs. A representative TEM microphotograph is presented in Figure 1, which shows that DEX-NPs have homogeneous spherical shapes and smooth surface. With respect to particle size, analyzed by PCS measurement, the DEX-NPs exhibited a narrow size distribution with an effective particle diameter of $232 \pm 5.4 \mathrm{~nm}$ and polydispersity index of 0.19 . The encapsulation efficiency of DEX in the nanoparticles was $56.0 \%$.

\section{In vitro release of DEX from PLGA nanoparticles}

The DEX release profile of nanoparticles has been investigated in detail with the dialysis membrane method. The cumulative release profiles of DEX were obtained by determining the percentage of DEX released with respect to the amount of DEX originally loaded in the nanoparticles (Figure 2).

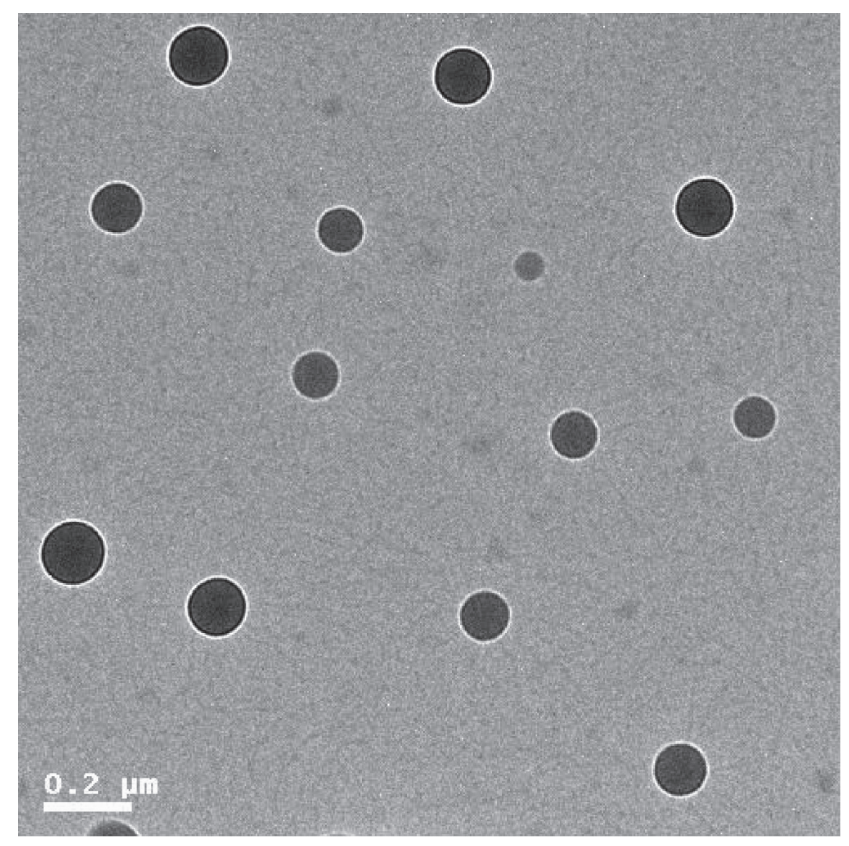

Figure I Transmission electronic microscopy image of dexamethasone-loaded PLGA nanoparticles (scale bar $=0.2 \mu \mathrm{m}$ ).

Abbreviation: PLGA, Poly(lactic acid-co-glycolic acid).

The in vitro drug release of the nanoparticles showed a continuous release for about 40 days without any lag time. The release profile showed almost no initial burst release, with about $6 \%$ release during the first day, followed by a constant release up to 35 days, during which more than $97 \%$ of the drug was released. The release rate appeared to have a linear or zero order release rate from day 2 to 35 . As the drug was depleted, the DEX release rate from the nanoparticles declined. The cumulative release of DEX after 40 days was

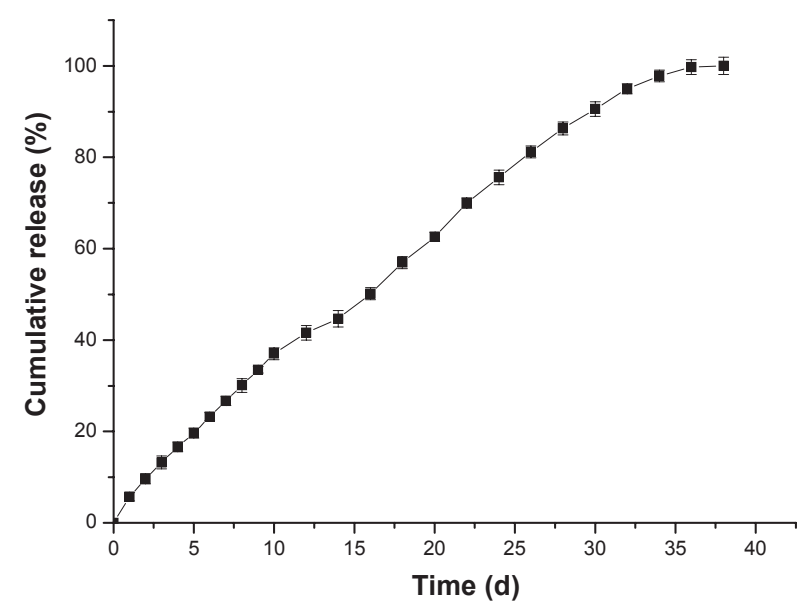

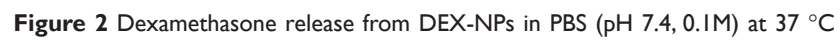
(mean $\pm \mathrm{SD}, \mathrm{n}=3$ ).

Abbreviations: DEX-NPs, dexamethasone-loaded PLGA nanoparticles; PBS, phosphate-buffered saline; PLGA, Poly(lactic acid-co-glycolic acid); SD, standard deviation. 
around $99 \%$. From the sustained drug release profile, it can be clearly inferred that the drug was well dispersed in the polymer matrix, which allowed gradual and constant release of the drug without any apparent initial burst.

\section{Ophthalmic observation}

Ophthalmic investigations, including fundus observation and photography, IOP measurement, and B-scan ocular ultrasonography were performed at each time point. All the eyes were examined with the indirect ophthalmoscope. Photography of the fundus showed that DEX-NPs were suspended within intravitreal spaces, and that they gradually sedimented to the vicinity of retina. The volume of nanoparticles decreased with time (Figure 3). There was no evidence of retinal abnormalities by indirect ophthalmoscopy.

IOP measurement in the rabbit eye was carried out using Schiötz tonometer. This instrument measures IOP by registering the depth of indentation produced in the cornea by a plunger carrying a known weight. At each time point, IOP stabilized between $8 \sim 20 \mathrm{~mm} \mathrm{Hg}$, and the mean IOP of the experimental group was $15 \pm 1 \mathrm{~mm} \mathrm{Hg}$. The Student's
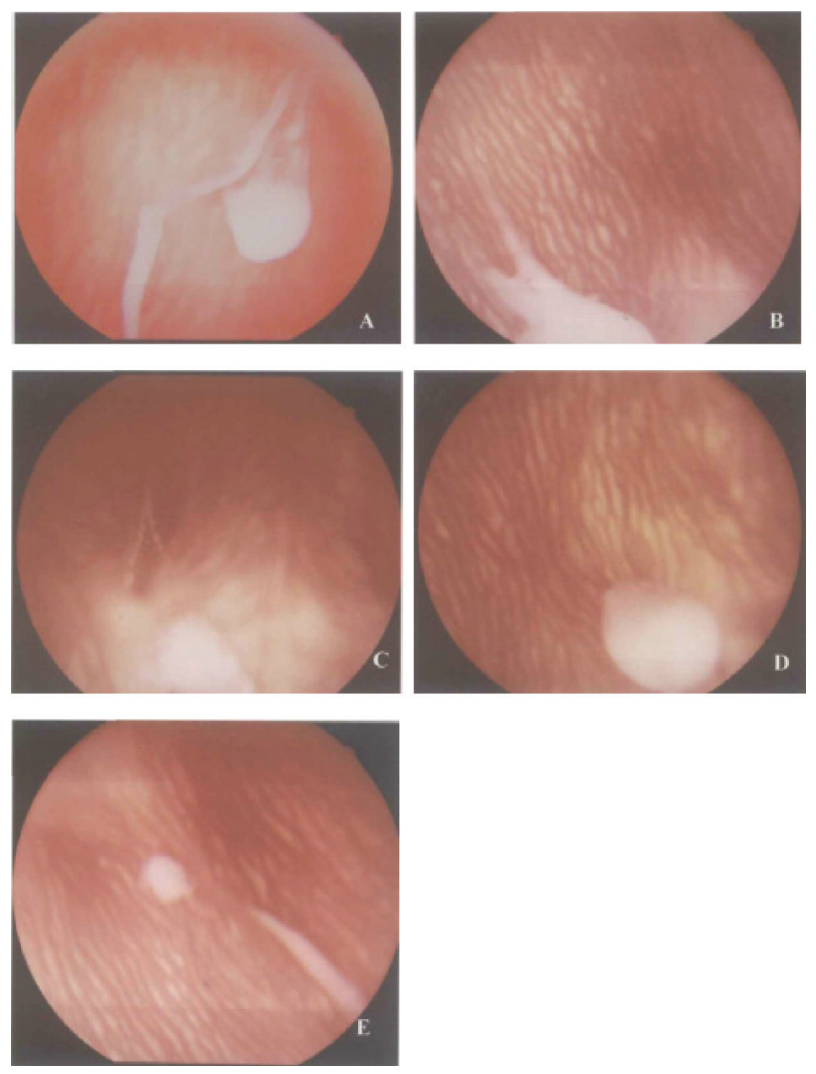

Figure 3 Fundus photograph of rabbit eye at (A) I day, (B) 7 days, (C) 21 days, (D) 35 days, and (E) 50 days after intravitreal injection of DEX-NPs.

Abbreviations: DEX-NPs, dexamethasone-loaded PLGA nanoparticles; PLGA, Poly(lactic acid-co-glycolic acid). $t$-test showed that there was no significant difference between the mean IOP values of the experimental group and the control group at any postoperative period, indicating that intravitreous injection and continuous release of DEX-NPs to rabbit eyes were not associated with IOP elevation.

B-scan ocular ultrasonography studies showed irregular middle-level echoes localized on the section behind the vitreous body and under the nose. These echoes were not consistent with the hemorrhagic echoes, and are most likely the result of the drug sedimentation. No other abnormal echo was found by ultrasonography.

\section{Pharmacokinetics}

The DEX concentration of all the samples was determined by HPLC at each time point (Tables 1 and 2). The DEX concentration in cornea, aqueous humor, and lens were all below the detectable limit $\left(10 \mu \mathrm{g} / \mathrm{L}^{-1}\right)$ of the HPLC assay, and could only be detected in the iris on the first day after injection, with a low value of $0.08 \pm 0.02 \mathrm{~g} / \mathrm{g}^{-1}$. The DEX-loaded nanoparticles maintained a sustained release of DEX for at least 50 days in the vitreous with a maximum concentration $\left(\mathrm{C}_{\max }\right)$ of $8.48 \pm 1.19 \mathrm{mg} / \mathrm{L}^{-1}$, and provided relatively constant DEX levels for more than 30 days. In contrast, in the control group treated with $\mathrm{DEX}$, the $\mathrm{C}_{\max }$ in the vitreous was $10.73 \pm 5.43 \mathrm{mg} / \mathrm{L}^{-1}$, with only trace amounts of DEX being detected on the 7th day after injection, and the DEX concentration dropping below the detectable limit $\left(10 \mu \mathrm{g} / \mathrm{L}^{-1}\right)$ before the 14 th day after injection (Figure 4$)$. The vitreous DEX concentration in the experimental animals treated with DEX-NPs was higher than that of the plasma, with mean vitreous DEX concentration of $3.85 \mathrm{mg} / \mathrm{L}^{-1}$, which was significantly higher $(P<0.001)$ than that of the plasma $\left(0.7 \mathrm{mg} / \mathrm{L}^{-1}\right)$ (Figure 5). Comparing the experimental group and the control group, the drug concentrations in the vitreous $(P=0.86)$, chorioretina $(P=0.74)$, and plasma $(P=0.156)$ showed no significant differences.

Area under the curve (AUC) is a reliable index to estimate the bioavailability of drugs. Calculation of areas under the curve revealed that the AUC of vitreous, chorioretina, and plasma in the experimental animals was 4.96, 4.15, and 6.35 times higher than that in the control animals, respectively, which indicates that the bioavailability of DEX in the experimental group (DEX-NPs) was greater than that in the control group (DEX) (Figure 6 and Table 3).

\section{Discussion}

Corticosteroids have been demonstrated to be efficient antiinflammatory drugs for the treatment of acute and chronic 
Table I Drug concentration in experimental group treated by intravitreal injection of DEX-loaded PLGA nanoparticles $(n=3)$

\begin{tabular}{|c|c|c|c|c|c|c|c|}
\hline \multirow[t]{2}{*}{ Time (d) } & \multicolumn{7}{|c|}{ Drug concentration (mean \pm SD) } \\
\hline & $\begin{array}{l}\text { Cornea } \\
\left(\mu g / g^{-1}\right)\end{array}$ & $\begin{array}{l}\text { Aqueous humor } \\
\left(\mathrm{mg} / \mathrm{L}^{-1}\right)\end{array}$ & $\begin{array}{l}\text { Lens } \\
\left(\mu g / g^{-1}\right)\end{array}$ & $\begin{array}{l}\text { Iris } \\
\left(\mu g / g^{-1}\right)\end{array}$ & $\begin{array}{l}\text { Vitreous } \\
\left(\mathrm{mg} / \mathrm{L}^{-1}\right)\end{array}$ & $\begin{array}{l}\text { Chorioretina } \\
\left(\mu \mathrm{g} / \mathrm{g}^{-1}\right)\end{array}$ & $\begin{array}{l}\text { Plasma } \\
\left(\mathrm{mg} / \mathrm{L}^{-1}\right)\end{array}$ \\
\hline 1 & - & - & - & $0.08 \pm 0.02$ & $8.48 \pm 1.19$ & $1.79 \pm 0.17$ & $0.23 \pm 0.06$ \\
\hline 3 & - & - & - & - & $4.78 \pm 2.44$ & $2.41 \pm 1.25$ & $0.23 \pm 0.01$ \\
\hline 7 & - & - & - & - & $3.11 \pm 0.58$ & $1.08 \pm 0.22$ & $|.02 \pm 0.3|$ \\
\hline 14 & - & - & - & - & $3.88 \pm 0.19$ & $1.96 \pm 0.01$ & $1.42 \pm 0.44$ \\
\hline 21 & - & - & - & - & $4.88 \pm 1.83$ & $1.08 \pm 0.12$ & $0.90 \pm 1.07$ \\
\hline 28 & - & - & - & - & $4.42 \pm 0.45$ & $0.99 \pm 0.02$ & $0.35 \pm 0.44$ \\
\hline 35 & - & - & - & - & $3.73 \pm 1.90$ & - & $0.68 \pm 0.07$ \\
\hline 42 & - & - & - & - & $2.73 \pm 1.32$ & - & $0.25 \pm 0.15$ \\
\hline 50 & - & - & - & - & $0.56 \pm 0.19$ & - & $0.75 \pm 1.36$ \\
\hline 80 & - & - & - & - & - & - & - \\
\hline
\end{tabular}

Abbreviations: DEX, dexamethasone; PLGA, Poly(lactic acid-co-glycolic acid); SD, standard deviation.

posterior segment eye diseases such as uveitis. ${ }^{13}$ Direct administration of drugs to the vitreous humor is frequently required to treat these eye diseases since a satisfactory access to retina through the blood vessels can not be expected when using a systemic route of administration. Intravitreal administration is an option to deliver the desired drug to its target tissue in the most direct fashion as well as preventing the adverse effects associated with the systemic treatment. Several acute and chronic posterior segment diseases are often treated with repeated intravitreal injections in order to maintain drug concentrations. However, multiple intraocular injections are poorly tolerated and have inherent risks. Therefore, the development of sustained release multiparticle system that can lead to a controlled drug release and are adapted to the intraocular route is valuable. Nanoparticle drug delivery offers advantages that allow a more targeted drug delivery and controllable release of the therapeutic compound. In this article, DEX-NPs were formulated and its pharmacokinetics as well as tolerance after intravitreal injection in rabbit eyes was evaluated. To our best knowledge, there have been no previous reports describing the pharmacokinetics and ocular tissue distribution of intravitreally injected DEX-NPs in rabbits.

DEX has been used frequently in both anterior- and posterior-segment treatments of the eye. The high potency and relatively acceptable toxicity of this compound, combined with its multifunctional roles as an anti-inflammatory, antiangiogenic, and antipermeability agent, confirm that it is efficient for ocular diseases. ${ }^{17-19}$ However, the pharmacokinetic half-life of DEX is $3 \mathrm{~h}$ which does not make it an ideal depot injection candidate for chronic conditions in the eye. ${ }^{15}$ This has been addressed in DEX-biodegradable intraocular implant (Posurdex; Allergan, Irvine, CA), which was designed to release DEX for an extended amount of time at a steady rate. It has proved to be effective to treat inflammation after cataract surgery, decreasing the

Table 2 Drug concentration of control animals treated by intravitreal injection of DEX $(n=3)$

\begin{tabular}{|c|c|c|c|c|c|c|c|}
\hline \multirow[t]{2}{*}{ Time (d) } & \multicolumn{7}{|c|}{ Drug concentration (mean \pm SD) } \\
\hline & $\begin{array}{l}\text { Cornea } \\
\left(\mu \mathrm{g} / \mathrm{g}^{-1}\right)\end{array}$ & $\begin{array}{l}\text { Aqueous humor } \\
\left(\mathrm{mg} / \mathrm{L}^{-1}\right)\end{array}$ & $\begin{array}{l}\text { Lens } \\
\left(\mu \mathrm{g} / \mathrm{g}^{-1}\right)\end{array}$ & $\begin{array}{l}\text { Iris } \\
\left(\mu g / g^{-1}\right)\end{array}$ & $\begin{array}{l}\text { Vitreous } \\
\left(\mathrm{mg} / \mathrm{L}^{-1}\right)\end{array}$ & $\begin{array}{l}\text { Chorioretina } \\
\left(\mu \mathrm{g} / \mathrm{g}^{-1}\right)\end{array}$ & $\begin{array}{l}\text { Plasma } \\
\left(\mathrm{mg} / \mathrm{L}^{-1}\right)\end{array}$ \\
\hline 1 & - & - & - & $0.21 \pm 0.06$ & $10.7 \pm 5.43$ & $2.46 \pm 1.67$ & $4.87 \pm 0.04$ \\
\hline 2 & - & - & - & - & $8.66 \pm 0.07$ & $1.47 \pm 0.21$ & $0.07 \pm 0.08$ \\
\hline 3 & - & - & - & - & $3.30 \pm 1.78$ & $1.73 \pm 0.46$ & $0.04 \pm 0.01$ \\
\hline 5 & - & - & - & - & $2.71 \pm 0.33$ & $1.26 \pm 0.18$ & $0.25 \pm 0.06$ \\
\hline 7 & - & - & - & - & $0.09 \pm 0.03$ & - & $0.90 \pm 0.07$ \\
\hline 14 & - & - & - & - & - & - & - \\
\hline
\end{tabular}

Abbreviations: DEX, dexamethasone; SD, standard deviation. 


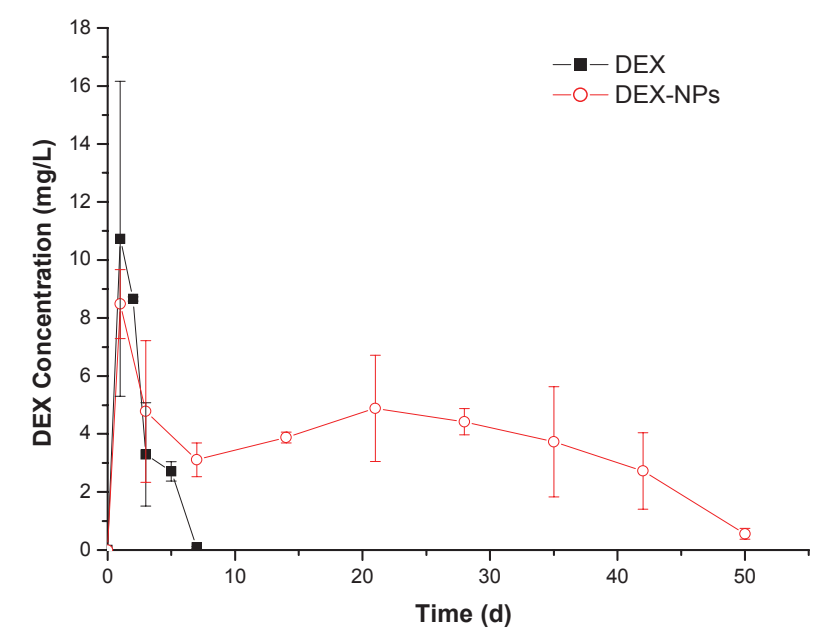

Figure 4 DEX concentrations in vitreous after intravitreal injection in rabbits of $(\boldsymbol{\square})$ DEX and $(O)$ DEX-NPs (mean $\pm S D, n=3$ ).

Abbreviations: DEX, dexamethasone; DEX-NPs, dexamethasone-loaded PLGA nanoparticles; PLGA, Poly(lactic acid-co-glycolic acid); SD, standard deviation.

risk of systemic toxicity and ocular side effects. ${ }^{20}$ Other ocular drug delivery systems currently under investigation for DEX include intraocular lens containing a DEX drug delivery system (IOL-DDS), ${ }^{19}$ an iontophoretic device assembled with DEX-loaded hydrogels ${ }^{17}$ and DEX nanoparticles. ${ }^{21}$

Drug-loaded polymeric nanoparticles (DNPs) has demonstrated promising results in ophthalmic drug delivery, offering favorable biological properties, such as biodegradability, nontoxicity, biocompatibility, and mucoadhesiveness. ${ }^{12}$ DNPs have been shown to be amenable to targeting of the drug to the site of action, leading to a decrease in the dose

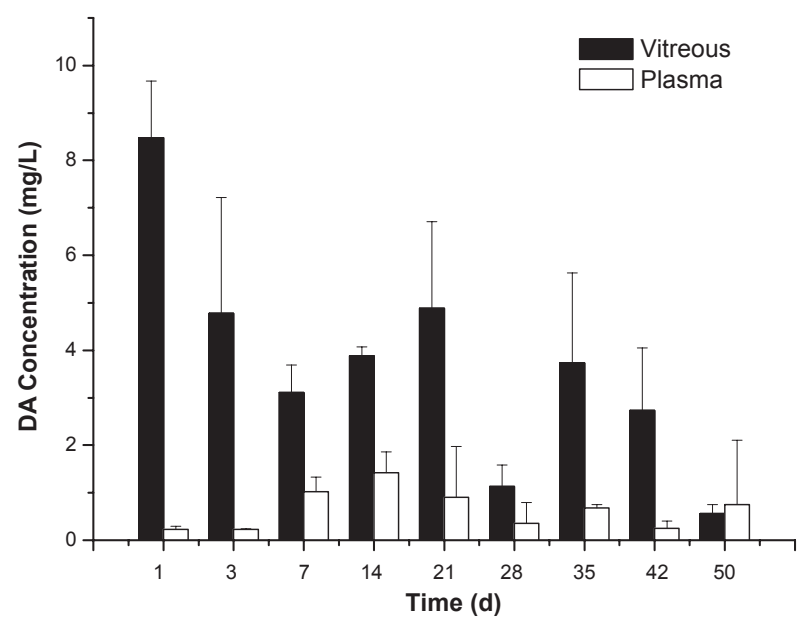

Figure 5 DEX concentrations in vitreous and plasma after intravitreal injection of DEX-NPs (mean \pm SD, $n=3$ ).

Abbreviations: DEX, dexamethasone; DEX-NPs, dexamethasone-loaded PLGA nanoparticles; PLGA, Poly(lactic acid-co-glycolic acid); SD, standard deviation.

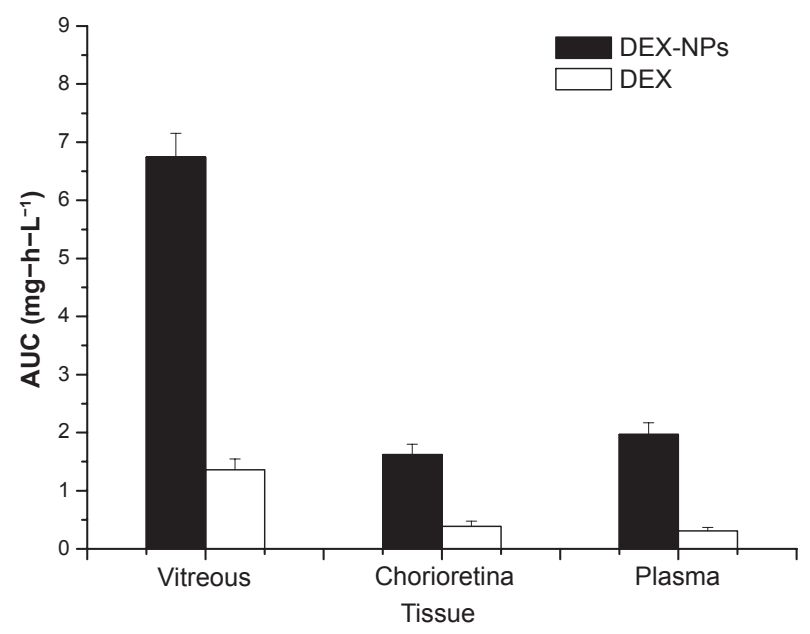

Figure 6 Area under the curve (AUC) of vitreous, chorioretina, and plasma after intravitreal injection of DEX-NPs and DEX (mean $\pm S D, n=3$ ).

Abbreviations: DEX, dexamethasone; DEX-NPs, dexamethasone-loaded PLGA nanoparticles; PLGA, Poly(lactic acid-co-glycolic acid); SD, standard deviation.

required and a decrease in side effects. DNPs can also be employed to achieve multiple purposes, including enhanced cellular uptake of poorly permeable drugs, reduced cellular and tissue clearance of drugs, and to sustain drug delivery over a desired time period. ${ }^{22-24}$ Furthermore, chief among the advantages mentioned above is the fact that no surgical procedures are necessary for implanting or removing the particles, in contrast to conventional large polymer implants. ${ }^{11}$ These properties are associated with better physical stability than liposomes and enable DNPs to fulfill the requirements for ophthalmic application. PLGA is typically used to formulate DNPs because it has a number of advantages over other polymers, including biodegradability, biocompatibility, and approval for human use by the US Food and Drug Administration (FDA). ${ }^{25-28}$ More importantly, the degradation rates of PLGA and the accompanying release of encapsulated drugs can be controlled by the polymer's physicochemical properties, such as molecular weight, hydrophilicity, and the ratio of lactide to glycolide..$^{29,30}$

Table 3 Area under the curve of vitreous, chorioretina, and plasma after intravitreal injection of DEX-NPs and DEX (mean \pm SD, $\mathrm{n}=3$ )

\begin{tabular}{llll}
\hline \multirow{2}{*}{ Group } & \multicolumn{3}{l}{ Area under the curve $\left(\mathrm{mg} / \mathrm{h} / \mathrm{L}^{-1}\right)$} \\
\cline { 2 - 4 } & Vitreous & Chorioretina & Plasma \\
\hline Experimental group & 6.74 & 1.62 & 1.97 \\
Control group & 1.36 & 0.39 & 0.31 \\
\hline
\end{tabular}

Abbreviations: DEX, dexamethasone; DEX-NPs, dexamethasone-loaded PLGA nanoparticles; PLGA, Poly(lactic acid-co-glycolic acid); SD, standard deviation. 
In this work, DEX-NPs were prepared using an emulsion/ solvent evaporation protocol, and were intravitreally injected in the rabbit eyes to investigate the feasibility of intraocular application of drug loaded nanoparticles. Intravitreally injected DEX-NPs bypass the blood-ocular barrier, allowing for the accumulation of higher intraocular drug concentrations than can be achieved by systemic or topical administration. In our work, the DEX-NPs demonstrated a prolonged presence following their intravitreal administration, and continued releasing DEX in the vitreous for at least 50 days, providing relatively constant drug levels in vitreous for more than one month with mean DEX concentration of $3.85 \mathrm{mg} / \mathrm{L}^{-1}$. Together, these factors maintained the effective level for suppressing inflammation $(0.15-4.00 \mu \mathrm{g} / \mathrm{ml})$ for more than a month following a single injection. ${ }^{31}$ The range of measurable intravitreal DEX concentrations in our study was higher than those reported in other studies, in which DEX delivery devices were implanted into the anterior chamber after cataract surgery. ${ }^{32-34}$ In addition, intravitreal injection is a far simpler procedure, and is likely to be associated with lower morbidity and cost compared to the implantation of a sustained delivery device. Furthermore, the DEX was concentrated in the vitreous and choroiretina, and the concentration in the vitreous was greater than that of plasma. Thus, the majority of the drug was absorbed by local tissue, while only small amount of it appeared in systemic system, which minimized the risk of systemic side effects. The calculation of AUC revealed that DEX bioavailability to ocular tissues was greatly improved by PLGA nanoparticles compared with regular DEX injection.

Intravitreal injection is frequently used for the diagnosis or treatment of many diseases of the posterior segment. It is a relatively easy technique to perform and is reliable, although slight variations in the injection site can provoke hemorrhage or inflammation. ${ }^{35}$ Intravitreal injection of corticosteroids in human ophthalmology is an increasingly common treatment for a variety of ophthalmic conditions. The sustained release systems are very useful for intraocular administration of drugs because with a single injection it would be possible to achieve the same effect as with multiple doses. In addition, they may fulfill the requirements of controlled degradation time and minimum inflammatory or toxic potential. In our studies, there were no abnormities that could be attributed to the intravitreal sustained-release DEX-NPs by clinical observation, including fundus observation and photography, IOP measurement and B-scan ocular ultrasonography. Marta and colleagues ${ }^{11}$ evaluated the ocular toxicity induced by the prolonged presence of ganciclovir-loaded bovine serum albumin nanoparticles after their intravitreal injection. They found the prolonged residence of nanoparticles in the eye seemed to be well tolerated and saw no inflammatory reactions or alterations in the tissue architecture. Therefore, they concluded that vision was not affected by autoimmune phenomena or alterations in the behavior of ophthalmic cells due to the intravitreal injection of nanoparticles. Study on inhibitory efficacy of intravitreal DEX acetate-loaded nanoparticles on choroidal neovascularization in a laser-induced rat model detected no sign of retinal toxity. ${ }^{21}$ Although the toxicity of DEX acetate-loaded PLGA nanoparticles to intraocular tissues was not directly determined in the present study, we believe that it is a relatively safe method of administering a prolonged therapeutic level of corticosteroid to intraocular tissues based upon our ophthalmologic observations.

\section{Conclusions}

For the first time, the pharmacokinetics, local distribution and tolerance of DEX-NPs have been investigated after intravitreal injection in the rabbit eyes. Intravitreal injection of DEX-NPs provides a more efficient means for improving the retention of the drug in the vitreal cavity, and allows for sustained release of DEX for at least 50 days in the rabbit eyes, during which relatively constant drug levels in the vitreous were obtained for about 30 days. Satisfactory biocompatibility and pharmacokinetic characters were obtained after intravitreal injection. DEX was concentrated in the vitreous and choroiretina, and the DEX concentration in the vitreous was far higher than that in the plasma, indicating the possibility of minimizing systemic side effects. To conclude, although further experiments are necessary, intravitreal injection of DEX-NPs shows great promise for the treatment of acute and chronic posterior segment eye diseases.

\section{Acknowledgments}

The authors thank and acknowledge financial support from Tianjin Natural Science Foundation of China (Contract No. 07JCYBJC01500) and Central Level, Nonprofit, Scientific Research Institutes' Basic R and D Operations Special Fund (Contract No. 0609). The authors report no conflicts of interest in this work.

\section{References}

1. Hughes PM, Olejnik O, Chang-Lin JE, et al. Topical and systemic drug delivery to the posterior segments. Adv Drug Deliv Rev. 2005; 57:2010-2032.

2. Geroski DH, Edelhauser HF. Drug delivery for posterior segment eye disease. Invest Ophthalmol Vis Sci. 2000;41:961-964.

3. Yasukawa T, Ogura Y, Sakurai E, et al. Intraocular sustained drug delivery using implantable polymeric devices. Adv Drug Deliv Rev. 2005;57:2033-2046. 
4. Martínez-Sancho C, Herrero-Vanrell R, Negro S. Vitamin A palmitate and aciclovir biodegradable microspheres for intraocular sustained release. Int J Pharm. 2006;326:100-106.

5. Moshfeghi AA, Peyman GA. Micro- and nanoparticulates. Adv Drug Deliv Rev. 2005;57:2047-2052.

6. Kathawate J, Acharya S. Computational modeling of intravitreal drug delivery in the vitreous chamber with different vitreous substitutes. Int $J$ Heat Mass Transfer. 2008;51:5598-5609.

7. Caspi RR, Chan CC, Leake WC, et al. Experimental autoimmune uveoretinitis in mice. Induction by a single eliciting event and dependence on quantitative parameters of immunization. J Autoimmun. 1990;3:237-246

8. Chan CC, Caspi RR, Ni M, et al. Pathology of experimental autoimmune uveoretinitis in mice. J Autoimmun. 1990;3:247-255.

9. De-Kozak Y, Mirshahi M, Boucheix C, et al. Prevention of experimental autoimmune uveoretinitis by active immunization with autoantigenspecific monoclonal antibodies. Eur J Immunol. 1987;17:541-547.

10. Schalken JJ, Winkens HJ, van Vugt AH, et al. Rhodopsin-induced experimental autoimmune uveoretinitis: dose-dependent clinicopathological features. Exp Eye Res. 1988;47:135-145.

11. Merodio M, Irache JM, Valamanesh F, et al. Ocular disposition and tolerance of ganciclovir-loaded albumin nanoparticles after intravitreal injection in rats. Biomaterials. 2002;23:1587-1594.

12. Sahoo SK, Dilnawaz F, Krishnakumar S. Nanotechnology in ocular drug delivery. Drug Discov Today. 2008;13:144-151.

13. Muñoz-Fernández S, Martín-Mola E. Uveitis. Best Pract Res Clin Rheumatol. 2006;20:487-505.

14. Gómez-Gaete C, Fattal E, Silva L, et al. Dexamethasone acetate encapsulation into Trojan particles. J Control Release. 2008;128:41-49

15. Hosseini K, Matsushima D, Johnson J, et al. Pharmacokinetic study of dexamethasone disodium phosphate using intravitreal, subconjunctival, and intravenous delivery routes in rabbits. J Ocul Pharmacol Ther. 2008;24:301-308.

16. Song CX, Labhasetwar V, Murphy H, et al. Formulation and characterization of biodegradable nanoparticles for intravascular local drug delivery. J Control Release. 1997;43:197-212.

17. Eljarrat-Binstock E, Raiskup F, Fruch-Pery J, et al. Transcorneal and trans-scleral iontophoresis of dexamethasone phosphate using drugloaded hydrogel. J Control Release. 2005;106:386-390.

18. Fialho SL, Behar-Cohen F, Silva-Cunha A. Dexamethasone-loaded poly(epsilon-caprolactone) intravitreal implants: A pilot study. Eur $J$ Pharm Biopharm. 2007;68:637-646.

19. Siqueira RC, Filho ER, Fialho SL, et al. Pharmacokinetic and toxicity investigations of a new intraocular lens with a dexamethasone drug delivery system: A pilot study. Ophthalmologica. 2006;220:338-342.

20. Hsu J. Drug delivery methods for posterior segment disease. Curr Opin Ophthalmol. 2007;18:235-239.

21. Xu J, Wang Y, Li Y, et al. Inhibitory efficacy of intravitreal dexamethasone acetate-loaded PLGA nanoparticles on choroidal neovascularization in a laser-induced rat model. $J$ Ocul Pharmacol Ther 2007;23:527-540
22. Tosi G, Costantino L, Ruozi B, et al. Polymeric nanoparticles for the drug delivery to the central nervous system. Expert Opin Drug Deliv. 2008;5:155-174.

23. Del-Amo EM, Urtti A. Current and future ophthalmic drug delivery systems. A shift to the posterior segment. Drug Discov Today. 2008;13: 135-143.

24. Booth BA, Vidal Denham L, Bouhanik S, et al. Sustained-release ophthalmic drug delivery systems for treatment of macular disorders: present and future applications. Drugs Aging. 2007;24:581-602.

25. Vega E, Gamisans F, García ML, et al. PLGA nanospheres for the ocular delivery of flurbiprofen: drug release and interactions. J Pharm Sci. 2008;97:5306-5317.

26. Mordenti J, Thomsen K, Licko V, et al. Intraocular pharmacokinetics and safety of a humanized monoclonal antibody in rabbits after intravitreal administration of a solution or a PLGA microsphere formulation. Toxicol Sci. 1999;52:101-106.

27. Gavini E, Chetoni P, Cossu M, et al. PLGA microspheres for the ocular delivery of a peptide drug, vancomycin using emulsification/ spray-drying as the preparation method:in vitro/in vivo studies. Eur J Pharm Biopharm. 2004;57:207-212.

28. Shive MS, Anderson JM. Biodegradation and biocompatibility of PLA and PLGA microspheres. Adv Drug Deliv Rev. 1997;28:5-24.

29. Jain RA, Rhodes CT, Railkar AM, et al. Controlled release of drugs from injectable in situ formed biodegradable PLGA microspheres: Effect of various formulation variables. Eur J Pharm Biopharm. 2000;50:257-262.

30. Duvvuri S, Janoria KG, Mitra AK. Development of a novel formulation containing poly(D,L-lactide-co-glycolide) microspheres dispersed in PLGA-PEG-PLGA gel for sustained delivery of ganciclovir. J Control Release. 2005;108:282-293.

31. Okabe K, Kimura H, Okabe J, et al. Intraocular tissue distribution of betamethasone after intrascleral administration using a nonbiodegradable sustained drug delivery device. Invest Ophthalmol Vis Sci. 2003;44:2702-2707.

32. Tan DT, Chee SP, Lim L, et al. Randomized clinical trial of Surodex steroid drug delivery system for cataract surgery: anterior versus posterior placement of two Surodex in the eye. Ophthalmology. 2001;108:2172-2181.

33. Tan DT, Chee SP, Lim L, et al. Randomized clinical trial of a new dexamethasone delivery system (Surodex) for treatment of postcataract surgery inflammation. Ophthalmology. 1999;106:223-231.

34. Wadood AC, Armbrecht AM, Aspinall PA, et al. Safety and efficacy of a dexamethasone anterior segment drug delivery system in patients after phacoemulsification. J Cataract Refract Surg. 2004;30: 761-768.

35. Molleda JM, Tardón RH, Gallardo JM, et al. The ocular effects of intravitreal triamcinolone acetonide in dogs. Vet J. 2008;176:326-332.
International Journal of Nanomedicine

\section{Publish your work in this journal}

The International Journal of Nanomedicine is an international, peerreviewed journal focusing on the application of nanotechnology in diagnostics, therapeutics, and drug delivery systems throughout the biomedical field. This journal is indexed on PubMed Central, MedLine, CAS, SciSearch $®$, Current Contents ${ } /$ Clinical Medicine,

\section{Dovepress}

Journal Citation Reports/Science Edition, EMBase, Scopus and the Elsevier Bibliographic databases. The manuscript management system is completely online and includes a very quick and fair peer-review system, which is all easy to use. Visit http://www.dovepress.com/ testimonials.php to read real quotes from published authors 